\title{
Information Flow and Cooperative Control of Vehicle Formations
}

\author{
J. Alexander Fax, Member, IEEE, and Richard M. Murray, Member, IEEE
}

\begin{abstract}
We consider the problem of cooperation among a collection of vehicles performing a shared task using intervehicle communication to coordinate their actions. Tools from algebraic graph theory prove useful in modeling the communication network and relating its topology to formation stability. We prove a Nyquist criterion that uses the eigenvalues of the graph Laplacian matrix to determine the effect of the communication topology on formation stability. We also propose a method for decentralized information exchange between vehicles. This approach realizes a dynamical system that supplies each vehicle with a common reference to be used for cooperative motion. We prove a separation principle that decomposes formation stability into two components: Stability of the is achieved information flow for the given graph and stability of an individual vehicle for the given controller. The information flow can thus be rendered highly robust to changes in the graph, enabling tight formation control despite limitations in intervehicle communication capability.
\end{abstract}

Index Terms-Cooperative control, graph theory, Laplacian, multivehicle control, stability.

\section{INTRODUCTION}

$\mathbf{R}$ ECENT technological advances have spurred a broad interest in autonomous, adaptable vehicle formations. The development of powerful control techniques for single vehicles, the explosion in computation and communication capabilities, and the advent of miniaturization technologies have elevated interest in vehicles which can interact autonomously with the environment and other vehicles to perform, in the presence of uncertainty and adversity, tasks beyond the ability of individual vehicles. Application areas include microsatellite clusters [1], [2], unmanned aerial vehicles (UAVs) [3], [4], autonomous underwater vehicles (AUVs) [5], [6], automated highway systems (AHSs) [7], [8], and mobile robotics [9], [10].

While each of these areas poses its own unique challenges, several common threads can be found. In most cases, the vehicles are coupled through the task they are trying to accomplish, but are otherwise dynamically decoupled, meaning the motion of one does not directly affect the others. Decisions must be made by each vehicle using only limited information about the

Manuscript received May 5, 2003; revised December 17, 2003. Recommended by Guest Editors P. Antsaklis and J. Baillieul. This work was supported by the Air Force Office of Scientific Research under Grants F49620-99-1-0190 and F49620-01-1-0460. The work of J. A. Fax was supported by a National Science Foundation Graduate Research Fellowship and an ARCS Foundation Fellowship.

J. A. Fax is with Northrop Grumman Electronic Systems, Woodland Hills, CA 91367 USA (e-mail: Alex.Fax@ngc.com).

R. M. Murray is with the Department of Control and Dynamical Systems, California Institute of Technology, Pasadena, CA 91125 USA (e-mail: murray@cds.caltech.edu).

Digital Object Identifier 10.1109/TAC.2004.834433 other vehicles, information that may be subject to uncertainty and transmission delay. The reaction of a vehicle to other vehicles' motions renders the formation an interconnected dynamical system whose behavior depends not only on the individual vehicle dynamics, but on the nature of their interconnection.

The cooperative behavior we focus on in this paper is formation control. Existing approaches to vehicle formation control generally fall into two cagetories. The "leader-follower" approach has the advantage of simplicity in that a reference trajectory is clearly defined by the leader, and in that internal stability of the formation is implied by stablity of the individual vehicles' control laws. However, leader-follower architectures are known to have poor disturbance rejection properties (see, e.g., [11]). Additionally, a leader-follower architecture depends heavily on the leader for achieving its goal, and over-reliance on a single vehicle in the formation may be undesirable, especially in adversarial environments. The second approach is the "virtual leader" approach [12], [6], in which vehicles in the formation jointly synthesize a single, possibly fictitious, leader vehicle whose trajectory acts as a leader for the group. This approach avoids the problems with disturbance rejection inherent in the leader-follower approach, but at the expense of high communication and computation capabities needed to synthesize the virtual leader and communicate its position in time to support real-time control of other vehicles.

What these approaches have in common is an assumption about the underlying communication topology that enables the use of a particular formation control methodology. We wish to consider a broader range of vehicle interconnection possibilities and understand how the topology of the information flow affects the stability and performance of the system as it performs a coordinated task. Our ultimate goal is the development of information exchange strategies which improve formation stability and performance and are robust to changes in the communication topology.

Our approach is to model the communication topologies as a graph; each vehicle is a vertex of a (directed) graph, and an arc is drawn from vertex $i$ to vertex $j$ if vehicle $i$ receives information from vehicle $j$. By merging ideas from graph theory and control theory, we are able to study the interplay between the communication network and vehicle dynamics, and to propose strategies for information exchange which mitigate those effects. We limit our focus to linear time-invariant (LTI) systems in order to elucidate the role of the graph in the system behavior. In that context, we provide necessary and sufficient conditions for stability of an interconnected system of indentical vehicles in terms of the eigenvalues of the graph Laplacian and show how to shape the information flow to achieve high performance. Portions of this work have been reported in [13]-[15]. 
The use of graphs in analysis of interconnected systems is not new. When decentralized control became an area of study in the 1970s, researchers used graph-theoretic ideas in modeling interconnections [16], [17]. The current broad interest in vehicle formations has revived an interest in graph-theoretic ideas [18]-[20]. A related area of research is synchronization of chaotic oscillators. References [21] and [22] identified the Laplacian eigenvalues as an important object of study. Other researchers took a more control-theoretic approach, using tools such as Lyapunov stability [23] and the circle criterion [24] to derive sufficient conditions for nonlinear chaotic oscillators to synchronize. The observation in [23] that the eigenvalues must be "negative enough" to achieve stability is similar is spirit to the work presented here. Our work differs in that we restrict our focus to stabilization of formations with linear dynamics, and as such we are able to make more precise statements about the role of Laplacian eigenvalues in determining formation stability. Our results are useful for controller design and also lay the foundation for our investigation of the role of intervehicle communication.

This paper is organized as follows. In Section II, we provide a brief summary of the relevant results in graph theory and define the relevant notation. The main stability results are derived in Section III, where we prove a Nyquist-like criterion for determining stability and explore the interaction between graph topology and vehicle dynamics through this criterion. In Section IV, we propose an information exchange methodology which is robust to uncertainty in the communication topology. This approach exhibits a separation principle which decouples the stability of the formation communication, which we term information flow, and the local control of individual vehicles. Finally, in Section V we summarize the main results and provide some thoughts on future directions of research.

\section{GRAPH THEORY}

Many excellent texts on graph theory exist; a recent example is [25]. Recent results regarding the Laplacian and its spectral structure can be found in [26]-[28]. The Perron-Frobenius theory can be found in many texts; the presentation here is based on material in [29]-[31].

\section{A. Definitions}

A directed graph $\mathcal{G}$ consists of a set of vertices, denoted $\mathcal{V}$, and a set of $\operatorname{arcs} \mathcal{A} \subset \mathcal{V}^{2}$, where $a=(\alpha, \beta) \in \mathcal{A}$ and $v, w \in$ $\mathcal{V}$. The first element of $a$ is denoted tail $(a)$, and the second is denoted head $(a)$. We assume that $\operatorname{tail}(a) \neq \operatorname{head}(a)$ for all $a$, meaning that the graph has no self-loops. We also assume that each element of $\mathcal{A}$ is unique. A graph with the property that for any $(\alpha, \beta) \in \mathcal{A}$, the arc $(\beta, \alpha) \in \mathcal{A}$ as well is said to be undirected. The in(out)-degree of a vertex $\alpha$, denoted $d_{i}(\alpha)$ $\left(d_{o}(\alpha)\right)$, is the number of arcs with $\alpha$ as its head (tail). If every possible arc exists, the graph is said to be complete.

A path on $\mathcal{G}$ of length $N$ from $\alpha_{0}$ to $\alpha_{N}$ is an ordered set of distinct vertices $\left\{\alpha_{0}, \ldots, \alpha_{N}\right\}$ such that $\left(\alpha_{i-1}, \alpha_{i}\right) \in \mathcal{A}$ for all $i \in[1, N]$. A graph in which a path exists from every vertex to every vertex is said to be strongly connected. A graph in which disjoint subsets of vertices exist that cannot be joined by any path is termed disconnected. An $N$-cycle on $\mathcal{G}$ is a path except for which $\alpha_{0}=\alpha_{N}$, meaning the path rejoins itself. A graph without cycles is said to be acyclic. A graph with the property that the set of all cycle lengths has a common divisor $k>1$ is said to be $k$-periodic.

The relationship between graph theory and control theory discussed in this paper makes use of matrices associated with a graph. For the purpose of defining these matrices, we assume that the vertices of $\mathcal{G}$ are enumerated, and each is denoted $\alpha_{i}$. The normalized adjacency matrix of a graph, denoted $G(\mathcal{G})$, is a square matrix of size $|\mathcal{V}|$, defined by $G_{i j}=1 / d_{o}\left(\alpha_{i}\right)$ if $\left(\alpha_{i}, \alpha_{j}\right) \in \mathcal{G}$, and is zero otherwise. When the graph in question is clear, this matrix will be denoted as $G$.

Following [28], we define the Laplacian of the graph as ${ }^{1}$

$$
L=I-G \text {. }
$$

It is possible to work with weighted graphs, in which the offdiagonal elements of $L$ are unequal yet still sum to -1 . Most of the results of the following sections do not depend on the arcs being weighted equally, though we will assume that for convenience. A sample graph and Laplacian are shown in Fig. 1.

Note that $G$ is nonnegative by construction, and that the rows of $L$ each sum to zero. The theory of nonnegative matrices will be quite useful in understanding the links between graph theory and vehicle formation control. Recall that a nonnegative matrix $G$ is termed irreducible if there does not exist a permutation matrix $P$ such that $P G P^{T}$ is block triangular, and is termed reducible otherwise. We will generally restrict our focus to irreducible matrices, though the structure of reducible matrices is useful in understanding the effects of multiple competing leaders in a formation; see [13]. Note that irreducibility implies strong connectivity. If $\mathcal{G}$ is aperiodic, then $G$ is termed primitive. If $\mathcal{G}$ is $k$-periodic, it is termed imprimitive, or cyclic of index $k$. We will denote the spectral radius of $G$ as $\rho(G)$. The following well-known theorem will be of use in our analysis.

Theorem 1 (Perron-Frobenius): Let $A$ be a nonnegative, irreducible matrix. The following are true:

1) $\rho(A)>0$;

2) $\rho(A)$ is a simple eigenvalue of $A$, and any eigenvalue of $A$ of the same modulus is also simple;

3) $A$ has a positive eigenvector $x$ corresponding to $\rho(A)$. Furthermore, if $A$ is primitive, then all eigenvalues of $A$ other than $\rho(A)$ have modulus strictly less than $\rho(A)$.

If $A$ is not primitive, the eigenvalues of $A$ have an interesting structure:

Theorem 2: Let $A$ be a nonnegative, irreducible matrix which is cyclic of index $k$. Then $A$ has $k$ eigenvalues of modulus $\rho(A)$, equal to $\lambda_{i}=\rho(A) e^{\frac{2 \pi j}{k} i}, i=0, \ldots, k-1$.

\section{B. Eigenvalues of Laplacians}

We now return to the structure of the spectrum of the Laplacian. The following can be shown to be true by observing that the rows of $L$ necessarily sum to zero, and that any eigenvalue $\lambda$ of $L$ corresponds to an eigenvalue $1-\lambda$ of $G$.

\footnotetext{
${ }^{1}$ Some references define $L$ as $D-A$, where $D$ is the matrix with the outdegrees along the diagonal and $A$ is the standard adjacency matrix. Others use the transpose of $A$ to define the Laplacian of the directed graph. This distinction is of little consequence in terms of the theory, but the definition stated above better suits our purposes.
} 


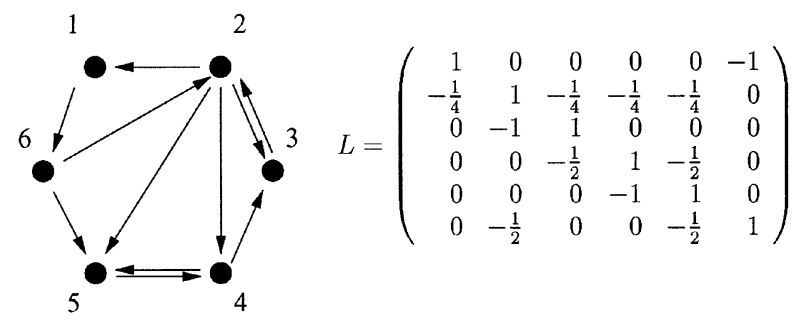

Fig. 1. Sample graph and Laplacian.

Proposition 1: Zero is an eigenvalue of $L$. The associated eigenvector is $1^{T}$.

Proposition 2: All eigenvalues of $L$ lie in a disk of radius 1 centered at the point $1+0 j$ in the complex plane.

Proposition 3: If $\mathcal{G}$ is strongly connected, the zero eigenvalue of $L$ is simple. If, in addition, $\mathcal{G}$ is aperiodic, all nonzero eigenvalues lie in the interior of the Perron disk. If $\mathcal{G}$ is $k$-periodic, $L$ has $k$ evenly spaced eigenvalues on the boundary of the Perron disk.

Proposition 4: If $\mathcal{G}$ is undirected, then all eigenvalues of $L$ are real.

\section{Kronecker Algebra and Formation Modeling}

A final tool that will be useful in modeling and maniuplating equations governing formation motion is Kronecker algebra. For example, if $\dot{x}_{i}=A x_{i}$ represents the dynamics of a single vehicle, the dynamics of $N$ vehicles can be represented as $\dot{x}=$ $\left(I_{N} \otimes A\right) x$. Another important case is if $A$ is an $N \times N$ matrix representing the manipulation of scalar data from $N$ vehicles, and that manipulation needs to be applied to each value of a vector of length $n$. In that case, the manipulation can be represented by concatenating the $N$ vectors of length $n$ into a single vector of length $N n$, and multiplying it by $A \otimes I_{n}$.

The associativity property of the Kronecker product facilitates manipulation of these matrices. In particular, if $X$ is an $r \times s$ matrix, and $Y$ is an $N \times N$ matrix, then

$$
\left(I_{N} \otimes X\right)\left(Y \otimes I_{s}\right)=\left(Y \otimes I_{r}\right)\left(I_{N} \otimes X\right)=Y \otimes X .
$$

\section{Relative Position CONTROL IN Vehicle Formations}

The problem we consider in this section is the stabilization of the relative position of a set of vehicles with identical linear dynamics.

\section{A. Formation Equations of Motion}

We consider a set of $N$ vehicles, whose (identical) linear dynamics are denoted

$$
\dot{x}_{i}=P_{A} x_{i}+P_{B} u_{i}
$$

where $x_{i} \in \mathbb{R}^{n}, u_{i} \in \mathbb{R}^{m}$ are the vehicle states and controls, and $i \in[1, N]$ is the index for the vehicles in the flock. Each vehicle receives the following measurements:

$$
\begin{aligned}
y_{i} & =P_{C_{1}} x_{i} \\
z_{i j} & =P_{C_{2}}\left(x_{i}-x_{j}\right), \quad j \in \mathcal{J}_{i}
\end{aligned}
$$

where the set $\mathcal{J}_{i} \subset[1, N] \backslash\{i\}$ represents the set of vehicles which vehicle $i$ can sense. Thus, $y_{i} \in \mathbb{R}^{k}$ represents internal state measurements, and $z_{i j} \in \mathbb{R}^{l}$ represents external state measurements relative to other vehicles. We assume that $\mathcal{J}_{i} \neq \emptyset$, meaning each vehicle can sense at least one other vehicle. Note that a single vehicle cannot drive all the $z_{i j}$ terms to zero simultaneously; the errors must be synthesized into a single signal. For simplicity, and without loss of generality, we assume that all relative state measurements are weighted equally to form one error measurement

$$
z_{i}=\frac{1}{\left|\mathcal{J}_{i}\right|} \sum_{j \in \mathcal{J}_{i}} z_{i j}
$$

where $\left|\mathcal{J}_{i}\right|$ is the cardinality of the set $\mathcal{J}_{i}$. The choice of weighting does not impact the results, as long as the weights for a given vehicle sum to one. We also define a decentralized control law $K$ which maps $y_{i}, z_{i}$ to $u_{i}$ and has internal states $v_{i} \in \mathbb{R}^{s}$, represented in state-space form by

$$
\begin{aligned}
\dot{v}_{i} & =K_{A} v_{i}+K_{B_{1}} y_{i}+K_{B_{2}} z_{i} \\
u_{i} & =K_{C} v_{i}+K_{D_{1}} y_{i}+K_{D_{2}} z_{i} .
\end{aligned}
$$

We now consider the system of all $N$ vehicles together, using the Kronecker product to assemble the matrices governing formation behavior, as discussed earlier. Let $x$ denote the concatenation of the vectors $x_{i}, \ldots, x_{N}$. Using this notation, the system dynamics are represented as follows:

$$
\left(\begin{array}{c}
\dot{x} \\
\dot{v}
\end{array}\right)=\left(\begin{array}{ll}
A_{11} & A_{12} \\
A_{21} & A_{22}
\end{array}\right)\left(\begin{array}{l}
x \\
v
\end{array}\right)
$$

where

$$
\begin{aligned}
A_{11}= & I_{N} \otimes\left(P_{A}+P_{B} K_{D_{1}} P_{C_{1}}\right) \\
& +\left(I_{N} \otimes P_{B} K_{D_{2}} P_{C_{2}}\right)\left(L \otimes I_{n}\right) \\
A_{12}= & I_{N} \otimes P_{B} K_{C} \\
A_{21}= & I_{N} \otimes K_{B_{1}} P_{C_{1}}+\left(I_{N} \otimes K_{B_{2}} P_{C_{2}}\right)\left(L \otimes I_{n}\right) \\
A_{22}= & I_{N} \otimes K_{A} .
\end{aligned}
$$

The matrix $L$ is defined as follows:

$$
\begin{aligned}
L_{i i} & =1 \\
L_{i j} & = \begin{cases}-\frac{1}{\left|\mathcal{J}_{i}\right|}, & j \in \mathcal{J}_{i} \\
0, & j \notin \mathcal{J}_{i} .\end{cases}
\end{aligned}
$$

and represents the relative sensing defined in (6). The Kronecker product is used to dimension $L$ to the size of the measurement vector, as discussed earlier.

\section{B. Formation Stability}

We are now able to identify the role of the sensing graph in the formation dynamics. The vehicles and their sensing indices $\mathcal{J}_{i}$ together form a graph, where each vertex represents a vehicle and an arc leads from vertex $i$ to vertex $j$ if $j \in \mathcal{J}_{i}$. Our assumption that each vehicle can sense at least one other vehicle implies that the out-degree of each vertex is at least 1 . The matrix $L$ defined in (9) is the Laplacian of the graph, defined in Section II-A. We show the following to be true. 
Theorem 3: A local controller $K$ stabilizes the formation dynamics in (8) iff it simultaneously stabilizes the set of $N$ systems

$$
\begin{aligned}
\dot{x}_{i} & =P_{A} x_{i}+P_{B} u_{i} \\
y_{i} & =P_{C_{1}} x_{i} \\
z_{i} & =\lambda_{i} P_{C_{2}} x_{i}
\end{aligned}
$$

where $\lambda_{i}$ are the eigenvalues of $L$.

Proof: We will show the above to be true by transforming the closed-loop dynamics in the following way. Let $T$ be a Schur transformation of $L$, meaning the unitary matrix such that $U=$ $T^{-1} L T$ is upper triangular with the eigenvalues of $L$ along the diagonal [30]. Clearly, $T \otimes I_{n}$ transforms $L \otimes I_{n}$ into $U \otimes I_{n}$. If we let $\tilde{x}=\left(T \otimes I_{n}\right) x$, and $\tilde{v}=\left(T \otimes I_{m}\right) v$, we can restate (8), using the associative property in (2), in terms of $\tilde{x}$ and $\tilde{v}$. The resulting matrix elements are

$$
\begin{aligned}
A_{11}= & I_{N} \otimes\left(P_{A}+P_{B} K_{D_{1}} P_{C_{1}}\right) \\
& +\left(I_{N} \otimes P_{B} K_{D_{2}} P_{C_{2}}\right)\left(U \otimes I_{n}\right) \\
A_{12}= & I_{N} \otimes P_{B} K_{C} \\
A_{21}= & I_{N} \otimes K_{B_{1}} P_{C_{1}}+\left(I_{N} \otimes K_{B_{2}} P_{C_{2}}\right)\left(U \otimes I_{n}\right) \\
A_{22}= & I_{N} \otimes K_{A} .
\end{aligned}
$$

Because the elements of the transformed system matrix are either block diagonal or block upper-triangular, the stability of this system is equivalent to the stability of the $N$ subsystems defined taking the diagonal blocks. For $U \otimes I_{n}$, the diagonal blocks are each $\lambda_{i} I_{n}$, so the $N$ diagonal subsystems can be written

$$
\begin{aligned}
& \dot{\tilde{x}}_{i}=\left(P_{A}+P_{B} K_{D_{1}} P_{C_{1}}+\lambda_{i} P_{B} K_{D_{2}} P_{C_{2}}\right) \tilde{x}_{i}+P_{B} K_{C} \tilde{v}_{i} \\
& \dot{\tilde{v}}_{i}=\left(K_{B_{1}} P_{C_{1}}+\lambda_{i} K_{B_{2}} P_{C_{2}}\right) \tilde{x}+K_{A} \tilde{v}
\end{aligned}
$$

which is equivalent to the controller $K$ stabilizing the system in (11).

Theorem 3 reveals that a formation of $N$ identical vehicles can be analyzed for stability by analyzing the stability of a single vehicle with the same dynamics, modified by only a scalar, representing the interconnection, that takes values according to the eigenvalues of the interconnection matrix $L$. This theorem applies to any matrix, not only a Laplacian. Note that $\lambda_{i}$ may be complex, leading to a complex-valued LTI in the previous formulation. The value in this formulation lies not in its direct use in design, but in its application to classical and modern stability robustness criteria, as we discuss later.

The zero eigenvalue of $L$ (see Proposition 1) can be interpreted as the unobservability of absolute motion of the formation in the measurements $z_{i}$. A prudent design strategy is to close an inner loop around $y_{i}$ such that the internal vehicle dynamics are stable, and then to close an outer loop around $z_{i}$ that achieves desired formation performance. For the remainder of this paper, we concern ourselves solely with the outer loop. Hence, we assume from now on that $P_{C_{1}}$ is empty and that $P_{A}$ has no eigenvalues in the open right-half plane. We do not wish to exclude eigenvalues along the $j \omega$ axis because they are characteristic of vehicle systems, representing the directions in which motion is possible. The controller $K$ is also presumed to be stable. If $K$ stabilizes the system in (11) for all $\lambda_{i}$ other than the zero eigenvalue, we say that it stabilizes the relative formation dynamics.
Note that for a strongly connected graph or for a formation with a single leader, it can be shown that the only equilibrium point is in fact the desired relative position of the vehicles. Additionally, the theorem is equally applicable when the inputs are driven not to zero, but to some internally consistent set of offsets [13].

Hereafter, we refer to the plant dynamics from $u_{i}$ to $y_{i}$ as $P$, its transfer function as $P(s)$, and that of the controller from $y_{i}$ to $u_{i}$ as $K(s)$. For single-input-single-output (SISO) systems, we can state a second version of Theorem 3 which is useful for stability and robustness analysis.

Theorem 4: Suppose $P$ is SISO. Then, $K$ stabilizes the relative formation dynamics iff the net encirclement of $-\lambda_{i}^{-1}$ by the Nyquist plot of $-K(s) P(s)$ is zero for all nonzero $\lambda_{i}$.

Proof: The Nyquist criterion states that stability of the closed-loop system in Theorem 3 is equivalent to the number of counterclockwise encirclements of $-1+j 0$ by the forward loop $\lambda_{i} P(j \omega) K(j \omega)$ being equal to the number of right-half plane poles of $P(s)$, which is assumed to be zero. This criterion is equivalent to the number of encirclements of $-\lambda_{i}^{-1}$ by $P(j \omega) K(j \omega)$ being zero.

In the case where $P$ is multiple-input-multiple-output (MIMO), the formation can be thought of as a structured uncertainty of the type scalar time identity (see [32]) where the scalars are the Laplacian eigenvalues. More specifically, we will write the eigenvalues as $\lambda_{i}=1+\mu_{i}$ and consider bounds on $\mu_{i}$. Suppose it is known that $\left|\mu_{i}\right| \leq M$ for all nonzero $\lambda_{i}$. If we close the loop around the unity block and leave $\mu_{i} I$ as an uncertainty, the resulting lower block is $C(s)=P(s) K(s)(I-P(s) K(s))^{-1}$, which is assumed to be stable. The following result from robust control theory then applies.

Theorem 5: $K$ stabilizes the relative formation dynamics of the MIMO vehicle $P$ if $\rho(C(j \omega))<M^{-1} \forall \omega \in(-\infty, \infty)$.

Example 1 (Double Integrator With Time Delay): Consider a system of the form $P(s)=\left(e^{-s T} / s^{2}\right)$, modeling a second order plant with time-delay, and suppose the plant has been stabilized with a PD control law. Fig. 2 shows a formation graph and the Nyquist plot of $K(s) P(s)$ with the Laplacian eigenvalues. The "o" locations correspond to the eigenvalues of the graph defined by the solid arcs in Fig. 2, and the "x" locations are for eigenvalues of the graph when the dashed arc is included as well. This example clearly shows the effect the formation has on stability margins. The standard Nyquist plot reveals a system with reasonable stability margins-about $8 \mathrm{~dB}$ and $45^{\circ}$. When one accounts for the effects of the formation, however, one sees that for the "o" formation, the stability margins are substantially degraded, and for the " $\mathrm{x}$ " formation, the system is in fact unstable. Interestingly, the formation is rendered unstable when additional information (its position relative to vehicle 6) is used by vehicle 1 . We will return to this point shortly.

\section{Evaluating Formations Via Laplacian Eigenvalues}

The location of Laplacian eigenvalues has emerged as the parameter which enables formation stability to be analyzed on the local level. We now turn to the question of bounding or predicting eigenvalue location based on properties of the graph. We begin by considering simple formation structures and their eigenvalue placement. Examples of these graphs are 

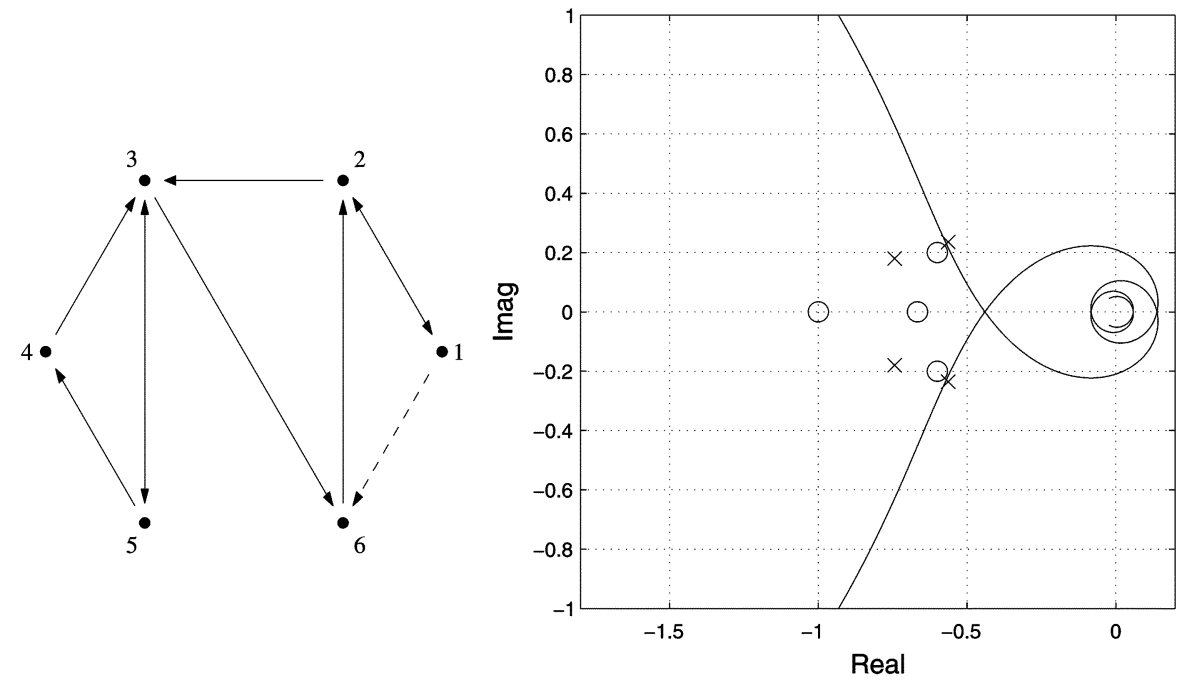

Fig. 2. Formation graph and Nyquist plot.

TABLE I

SAMPLE GRAPHS, SPECTRA, AND NYQUIST LOCATIONS

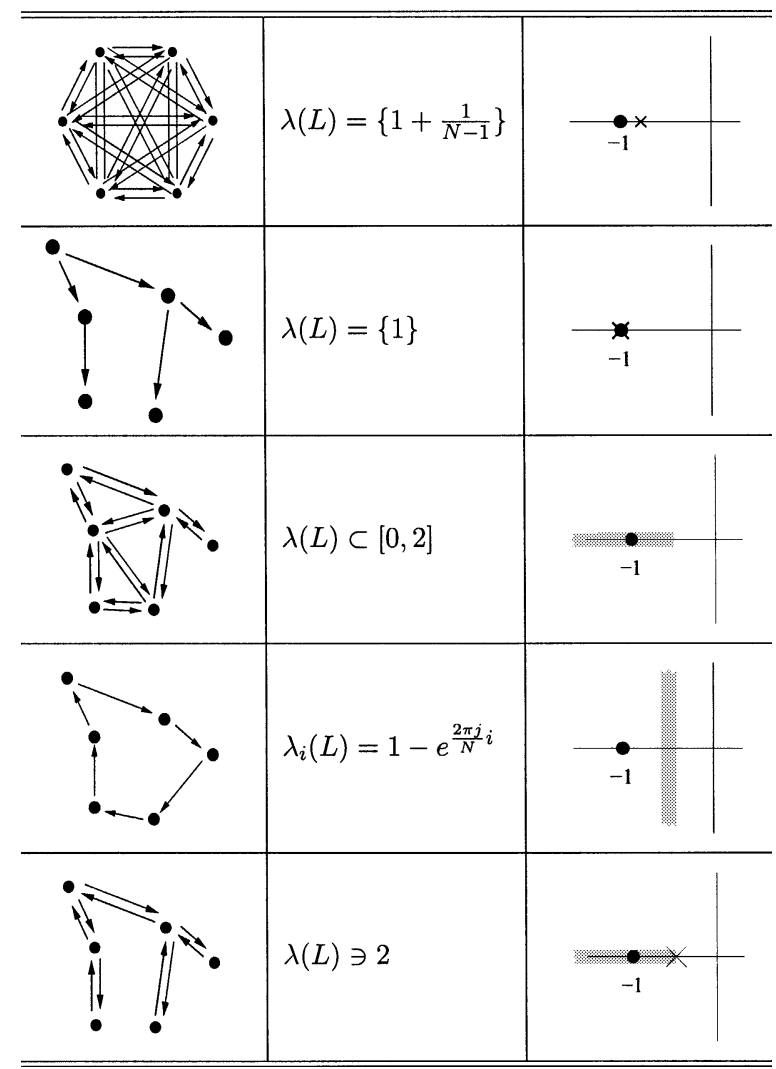

shown in Table I, where sample graphs, their nonzero spectra, and the locations on the Nyquist plot are shown.

1) Complete graph: The complete graph is one where every possible arc exists. In this case, the eigenvalues of a graph with $N$ vertices can be analytically determined to be zero and $1+1 /(N-1)$, the latter repeated $N-1$ times. For large $N$, stabilization of the complete graph is equivalent to stabilizing an individual vehicle.

2) Acyclic (directed) graph: This graph has the 1 eigenvalue repeated $N$ times. This can be seen from the fact that the vertices can be ordered such that $L$ is upper triangular with ones along the diagonal. This is the "leader-follower" architecture discussed earlier. In this case, vehicle stabilization is truly a local result, since other vehicles' dynamics enter only as a disturbance.

3) Single directed cycle: This graph is periodic and, therefore, has eigenvalues at $1-e^{j(i-1) / 2 \pi}, i \in[1, N]$ according to Proposition 3. These eigenvalues lie on the boundary of the Perron disk in which all the eigenvalues must lie. Note that the negative inverse of these points lie on the -0.5 vertical in the complex plane.

4) Two-cyclic undirected graph: A graph of this type would include a vehicle platoon with bidirectional position measurement. This graph will have an eigenvalue at 2 , due to its periodicity, and all other eigenvalues will be real, due to the symmetry of the graph.

Fig. 3 shows various eigenvalue regions for $-L$ and the corresponding regions for $-L^{-1}$. The region bounded by the solid line is the Perron disk in which all eigenvalues must lie. Its inverse is the LHP shifted by -0.5 . The dashed region is a bound in the magnitude of the nonzero eigenvalues of $L$. It corresponds to a shifted circle on the right-hand side of Fig. 3. Finally, the dashed-dotted line corresponds to a bound on the real component of the eigenvalues. The inverse of this bound corresponds to a circle which touches the origin. The shaded region represents the "desirable" region, in which the eigenvalues' locations do not differ substantially from -1 .

If we consider the complete graph and the single directed cycle graph of Table I as representing two extremes-one with all eigenvalues at a single location, the other with eigenvalues maximally dispersed, we see that eigenvalue placement can be related to the rate of mixing of information through the network. When the graph is highly connected, the global component of an individual vehicle's dynamics are rapidly averaged out through the rest of the graph, and thus has only a minor effect on stability. When the graph is periodic, the global component of the dynamics introduces periodic forcing of the vehicle, and the rest of the network never averages it out. This is represented on the Nyquist plot by putting the inverse eigenvalues nearer to the 

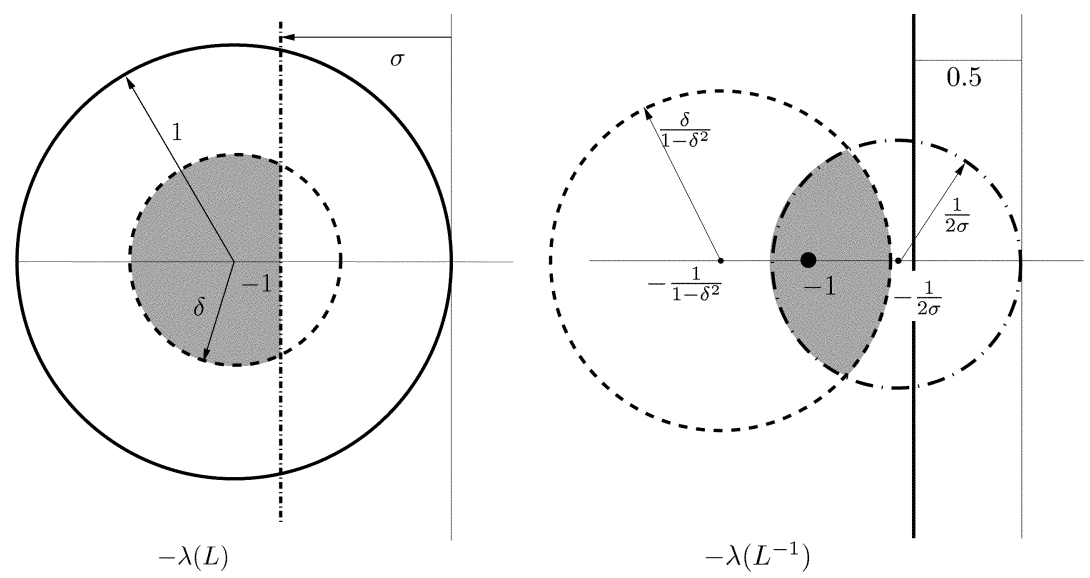

Fig. 3. Inclusion regions for $-\lambda(L)$ and $-\lambda(L)^{-1}$. The left figure shows various eigenvalue regions for $-L$ and the right figure shows the corresponding regions for $-L^{-1}$

imaginary axis, thus diminishing stability margins. This observation explains why the system in Fig. 2 loses stability margin when a link is added. The "solid" graph possesses two 3-cycles and two 2-cycles. When the dashed link is added, an additional 3 -cycle is created, rendering the graph more nearly 3-periodic. This drives two of the eigenvalues nearer to the positions they would occupy if the graph were truly periodic, i.e., the -0.5 vertical. The observations made in this section are qualitative; It would appear from here a productive line of research would be establishing measures of near-periodicity useful in quantifying formation stability margins.

\section{INFORMATION FlOW IN VEHICLE FORMATIONS}

In this section, we explore a paradigm for information exchange that enables the vehicles to jointly determine a virtual formation leader which does not presuppose any communication topology, and whose stabilty and performance are robust to uncertainties in the graph. The stability analysis tools developed in the previous section will be useful in this development, as will theorems from Perron-Frobenius theory reviewed in Section II-A.

In the previous section, we assume that sensed information was available instantaneously, and we used a continuous-time model of the vehicle dynamics. In this section, we assume that information takes a fixed time $T$ to travel between vehicles. To facilitate analysis, we also model each vehicle's dynamics $P$ as a discrete-time dynamical system

$$
\begin{aligned}
x_{k+1}^{i} & =P_{A} x_{k}^{i}+P_{B} u_{k}^{i} \\
y_{k}^{i} & =P_{C} x_{k}^{i}+P_{D} u_{k}^{i}
\end{aligned}
$$

where $k$ is the time step of duration $T$ and $i$ is the vehicle index. The error signal is given in (6). The controller $K$ is redefined similarly. Note that the stability results of Section III-B can be reproduced for discrete time systems by plotting the response of the discrete-time transfer function for $z=e^{j \omega}$ and applying the Nyquist criterion.

Broadly speaking, any decentralized formation control system consists of vehicles receiving a transmission from other vehicles, performing some computation using available information, transmitting the results of that computation, and using it as the basis for control. We can view this process as a discrete-time dynamical system where the states $p_{i}$ are the information at each vehicle. Our approach to the use of information is to shape this dynamical system to ensure that its evolution has the desired stability and convergence properties. We term this approach information flow.

The block diagram representing information flow is shown in Fig. 4 for the case where relative measurements $z$ are scalar. The method applies equally to vector measurements, which can be incorporated using the Kronecker product, as shown earlier but emitted here for clarity. The Laplacian continues to represent the sensing of relative position. Absolute vehicle offsets $h_{0}$ are included, and a consistent set of commanded relative offsets are defined by passing $h_{0}$ through $L$. In this case, $G$ represents the transmission and averaging of information, denoted $p$, whose dimension is the same as $y$. The graph representing communication topology is presumed to coincide with the sensing graph. The "information" $p$, whose role will be elucidated, is the output of a filter $I_{N} \otimes R$, which represents each vehicle's local computation. The inputs to each vehicle's information flow law are the averaged sensed errors and the averaged transmitted information, represented by $G$ in the feedback path. We constrain $R$ to be strictly proper, to account for one time step of sensing and transmission delay. Finally, the information filter is augmented by the output of a predictor $\tilde{P}$, ideally is equal to $P$, whose output is filtered by $H$ to produce the feedforward correction term $w$, also dimensioned compatibly with $y$.

\section{A. Properties of the Information Flow Loop}

To understand this approach, we first examine properties of the information flow filter when decoupled from the vehicle control and predictor. To understand the convergence of this loop, we first look at the simplest case, namely $R(z)=(1 / z)$, which can be written as

$$
p_{k+1}^{i}=\frac{1}{\left|\mathcal{J}_{i}\right|} \sum_{j \in \mathcal{J}_{i}} p_{k}^{j}+\left(y_{k}^{i}-y_{k}^{j}\right)
$$

or, in vector form, $p_{k+1}=G p_{k}+L y_{k}$. This system is neutrally stable due to the Perron root of $G$. The following theorem describes the evolution of this system.

Theorem 6: Suppose the directed graph $\mathcal{G}(G)$ is strongly connected and aperiodic, and let the input $y_{k}$ be constant. The 


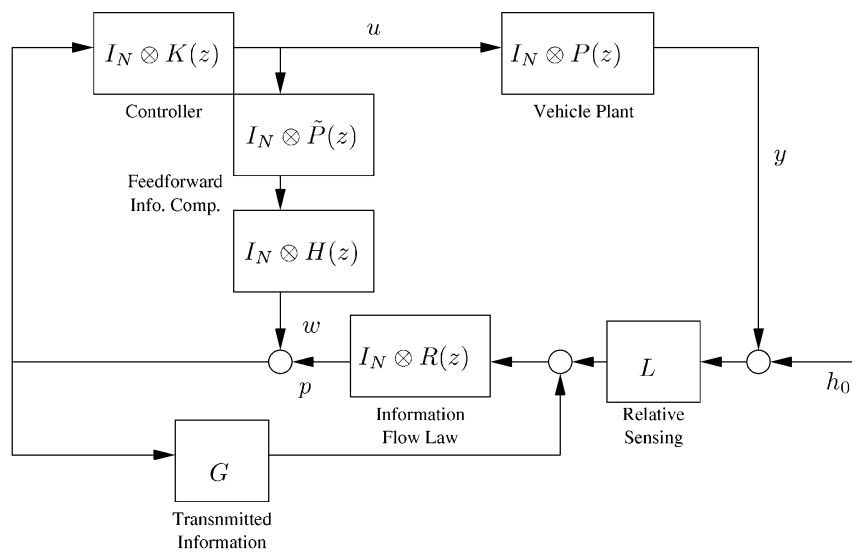

Fig. 4. Block diagram of information flow in the loop.

steady state value of the dynamical system in (15), when $p_{0}=0$, is

$$
p_{\infty}^{i}=y^{i}-\sum_{j=1}^{N} e_{l}^{j} y^{j}
$$

where $e_{l}^{i}$ is the $i$ th element of the left Perron eigenvector of $G$, scaled so that $\sum e_{l}^{i}=1$.

Proof: Let $e_{r}$ denote the right Perron eigenvector of $G$, and $e_{l}$ its left Perron eigenvector, normalized such that $e_{r}^{T} e_{l}=$ 1. If $G$ is irreducible, both $e_{r}$ and $e_{l}$ are positive (Theorem 1), so such a scaling must exist. Let $E=e_{r} e_{l}^{T}$. The following relationships between $G$ and $E$ are known to be true (see [30, p. 498] and recall that the Perron eigenvalue is 1).

Lemma 7: $G^{j}=E+(G-E)^{j}$.

Lemma 8: The eigenvalues of $G-E$ are the eigenvalues of $G$ with the Perron eigenvalue replaced with a zero eigenvalue.

Now, our system evolves as follows for $k>0$ :

$$
p_{k}=G^{k} p_{0}+\left(\sum_{j=0}^{k-1} G^{j}\right) L y .
$$

We assume that $p_{0}=0$, and we wish to find $p_{s s}=\lim _{k \rightarrow \infty} p_{k}$, if such a limit exists. Substituting into (17) via Lemma 7, we have

$$
p_{k}=\left(\sum_{j=0}^{k-1} E^{j}+(G-E)^{j}\right) L y .
$$

Recalling that $E=e_{r} e_{l}^{T}$, and that $L$ shares eigenvectors with $G$, we see that $e_{r}$ and $e_{l}$ are the eigenvectors of $L$ corresponding to the zero eigenvector. Therefore, $E L=e_{r} e_{l}^{T} L=e_{r} 0=0$, and the $E^{j}$ term can be eliminated from (18). Because $G$ is assumed irreducible and aperiodic, all non-Perron eigenvalues of $G$ have modulus strictly less than one (Theorem 3 ). Therefore, by Lemma 8, we see that $\rho(G-E)<1$. The infinite expansion of $p_{\infty}$ therefore converges [30, p. 301] and can be written as $p_{\infty}=(I-G+E)^{-1} L y=(L+E)^{-1} L y=$ $(L+E)^{-1}(L+E-E) y=\left(I-(L+E)^{-1} E\right) y$. Now, $L e_{r}=0$, and $E e_{r}=\left(e_{r} e_{l}^{T}\right) e_{r}=e_{r}\left(e_{l}^{T} e_{r}\right)=e_{r}$, so $(L+E) e_{r}=e_{r} \Rightarrow$ $(L+E)^{-1} e_{r}=e_{r}$, and hence $p_{\infty}=\left(I-(L+E)^{-1} e_{r} e_{l}^{T}\right) y=$ $\left(I-e_{r} e_{l}^{T}\right) y=(I-E) y$. The eigenvector $e_{r}$ is known to be $1^{T}$. The eigenvector $e_{l}$ is positive, and is scaled such that $\sum e_{l}^{i}=1$.
The columns of $E$ are, therefore, constant, and the rows are each $e_{l}^{T}$. Therefore, $p_{\infty}$ can be written component-wise as

$$
p_{\infty}^{i}=y^{i}-\sum_{j=1}^{N} e_{l}^{j} y^{j} .
$$

The information flow loop, therefore, has the effect of having the formation track the formation center, where the center is defined according to a weighting given by the graph. The key is that the formation center, given by $\sum_{j=1}^{N} e_{l}^{j} y^{j}$ in (19), is common to all vehicles, indicating that the formation has reached consensus as to its center. In this architecture, the weighting that defines the center cannot be chosen, though in principle it could be set by unevenly weighting the information when performing the averaging. However, this would require global knowledge of the graph, which is assumed not to be available.

This approach can be expanded to accommodate a more general information flow law that enables the designer to influence the dynamics of the information flow

$$
\begin{aligned}
q_{k+1} & =\sum_{j=0}^{M} a_{j} q_{k-j}+G p_{k}+L y_{k} \\
p_{k} & =\sum_{j=0}^{M} b_{j} q_{k-j} .
\end{aligned}
$$

For which $R(z)=\left(\sum_{j=0}^{M} b_{j} z^{M-j}\right) /\left(z^{M+1}-\sum_{j=0}^{M} a_{j} z^{M-j}\right)$ In this case, we prove separate stability and convergence theorems.

Theorem 9: The system in (20) is (neutrally) stable iff the transfer function

$$
F(z)=\frac{R(z)}{1-R(z)}
$$

is (neutrally) stable and its Nyquist plot avoids encirclement of the negative inverse of any of the nonzero eigenvalues of $L$.

Proof: Equation (20) can be rewritten as

$$
p(z)=\left(I_{N} \otimes R(z)\right)(G p(z)+L y(z))
$$

which can be transformed to the form

$$
p(z)=\left(I_{N} \otimes F(z)\right) L(-p(z)+y(z)) .
$$

This equation has the form of a feedback loop with $I_{N} \otimes F(z)$ in the forward path and $L$ in the reverse, and is therefore subject to the same stability analysis used for vehicle formations in Section III, where it was shown in Theorems 3 and 4 that the stability of this system is given by the Nyquist criterion stated above. Because one set of eigenvalues of this system corresponds to the open-loop dynamics, this system can be at best neutrally stable if $F(z)$ is itself neutrally stable.

We now turn to the steady-state performance of the information flow law. We assume that $F(z)$ has all poles on the interior of the unit circle with the possible exception of a simple pole at 1 , and that the polynomial $\sum_{i=0}^{M} a_{i} z^{M-i}$ has roots in the interior of the unit circle.

Theorem 10: If $F(z)$ stabilizes $L$ in the sense of Theorem 9, and under the previous assumptions, then the information flow law converges to the value

$$
p_{\infty}=c\left(I-c E-(1-c)(I-c(G-E))^{-1} G\right) y
$$
where $a=\sum_{j=0}^{M} a_{j}, b=\sum_{j=0}^{M} b_{j}$, and $c=(b / 1-a)$. 


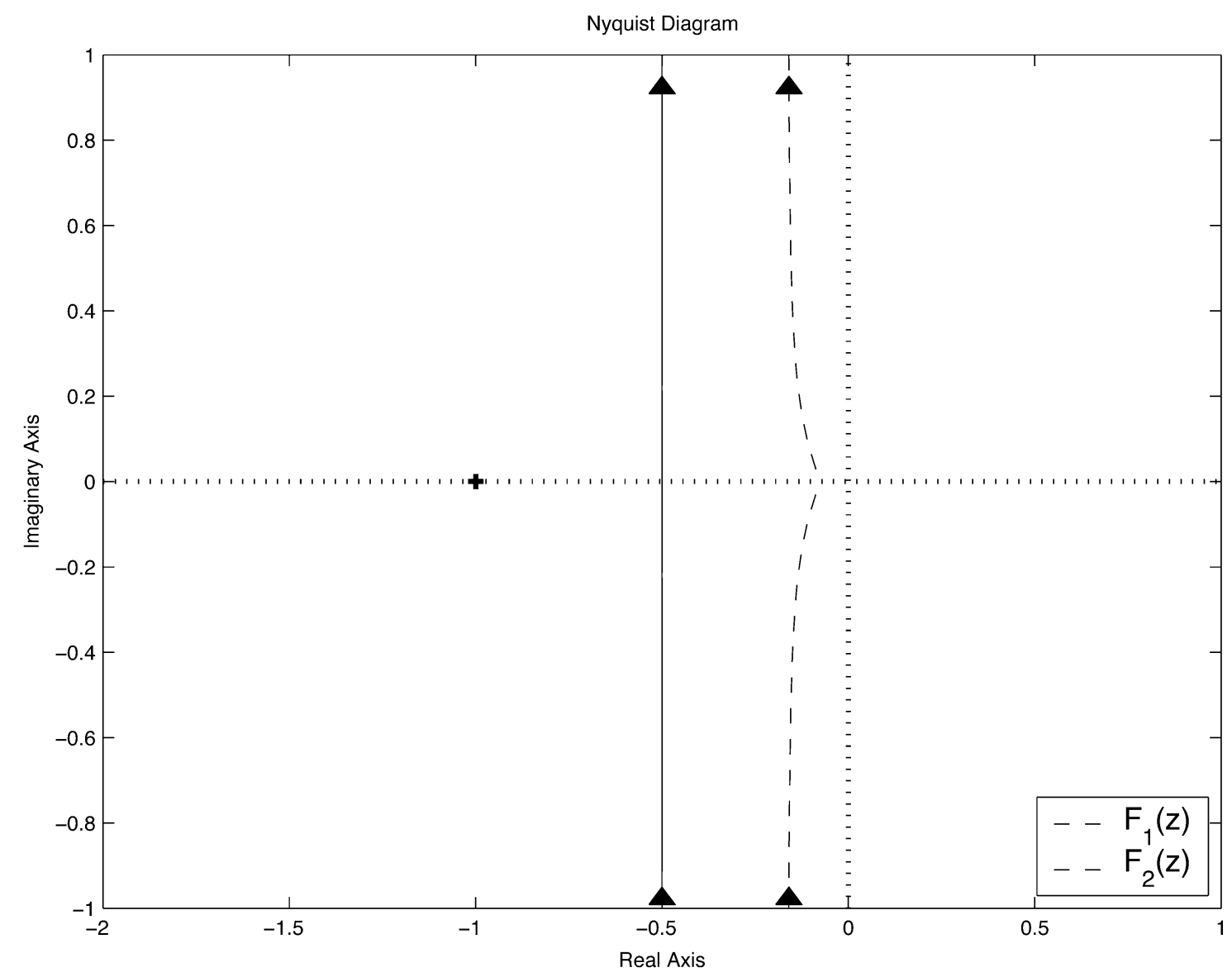

Fig. 5. Information flow filter Nyquist plots.

Proof: The proof is a lengthier version of the proof for Theorem 6, and can be found in [13].

Noting that the denominator of $F(z)$ is equal to $z^{M+1}-$ $\sum_{j=0}^{M}\left(a_{j}+b_{j}\right) z^{M-j}$, and that $c=1$ corresponds to $a+b=1$, we see that $c=1$ implies that $F(z)$ has a pole at 1 . When $c=1$, we recover the steady-state result of Theorem 6 , only we now see it to be true for any information flow filter with a pole at 1 (and which stabilizes the graph). When $c<1$, the steady-state is offset by an additional term. Note that when $c=1$, the vehicles all agree on the location of the formation center (expressed in each vehicle's coordinates), while when $c<1$, they do not. We can say that when $c=1$, the vehicles achieve consensus on formation center. From this perspective, having $c=1$ appears to be a desirable property of the information flow filter. However, when $c=1$, the system is only neutrally stable. The reason the filter converges to a steady state is because the input passed through $L$, whose kernel is equal to the Perron eigenvector of $G$. However, the presence of noise or sensor errors has the potential to introduce drift. Additionally, the eigenvalue at 1 means that old information never decays out, rendering the system sensitive to initial conditions. Of course, the initial conditions of the information flow law can be set (or reset) by the vehicle, assuming the existence of a protocol which guaranteed that this could be done without disrupting the formation.

Example 2: To understand the effects of shaping the information flow, we consider two examples. The first filter is in (15). In this case, following (21), $F_{1}(z)=1 /(z-1)$. The second filter is $F_{2}(z)=0.1875(z-0.1) /((z-0.25)(z-1))$. The pole at 1 means that $c=1$ in both cases. Fig. 5 shows the Nyquist plot for these two filters and Fig. 6 shows the convergence of the systems to constant input. The first lies along the -0.5 vertical. Points on that line correspond to periodic graphs (see Section II-B), which confirms Theorem 6. The second lies entirely to the right of the -0.5 vertical. Recalling from Section III-C that the Nyquist plot points generated by the Laplacian, $-\lambda_{i}(L)^{-1}$, all must lie on or to the left of this vertical, we conclude that this information flow law stabilizes any graph. While both settle in approximately the same time, the first filter exhibits ringing due to the proximity of the closed loop poles to the unit circle. The second filter has a much smoother response. We see how the information flow filter can be designed to achieve desirable responses and robustness to uncertainty in the graph.

\section{B. Information Flow in the Loop}

The information flow filter supplies each vehicle with the information it cannot sense: a formation center to use as a reference for control. The information $p$ represents the position of the virtual leader relative to each vehicle, and is therefore the logical input to the controller $K$. We can analyze the stability of this architecture, shown in Fig. 4 (with the predictor still disabled), by isolating $L$ and applying the Nyquist criterion as in Theorem 4 . In this case, one determines stability by analyzing the Nyquist plot of $F(z)(1+K(z) P(z))$. For a given plant and controller, the information flow loop can be designed to provide 


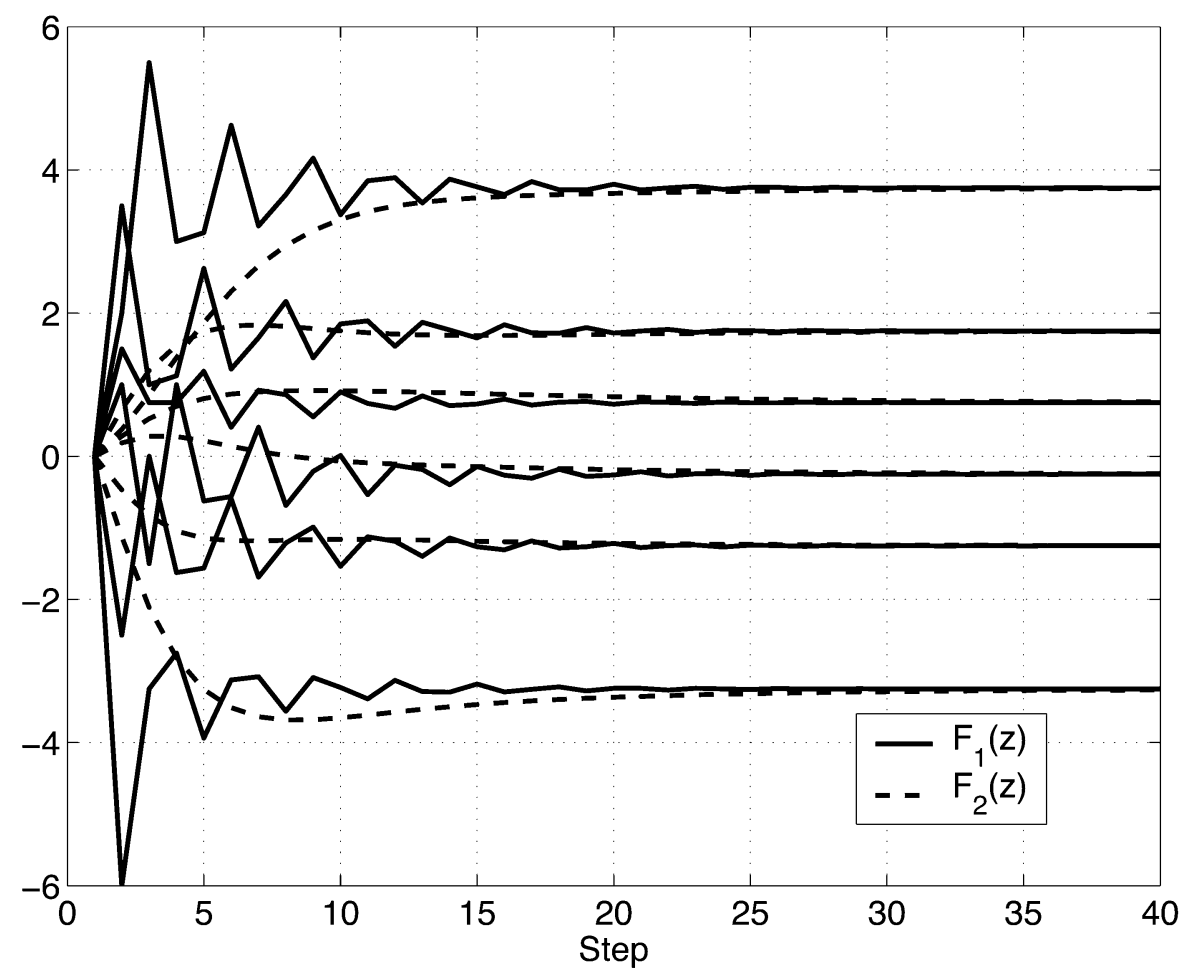

Fig. 6. Information flow filter convergence.

desirable margins. However, care must be taken in interpreting the stability margins derived from this plot. The gain and phase margins of this plot do not correspond to uncertainties in the plant in the typical fashion due to the location of $P$ in the transfer function.

To improve upon this, we turn to the predictor. In the absence of the predictor, the information flow loop is necessarily reactive to vehicle motions. When the predictor term is included, the information flaw law has the ability to correct the information it transmits based on its own expected motion. We realize this by adding $w_{i}$, the output of the filtered predictor, as a feedforward term to $p_{i}$, the output of each vehicle's information flow law, prior to transmission or use as an error signal for control. This architecture can be seen in Fig. 4. When $H(z)$ is chosen properly, the following result can be derived.

Theorem 11: Choose $H(z)$ to be

$$
H(z)=1-R(z)
$$

and suppose the feedback interconnection of $P(z)$ and $K(z)$ is well-posed. Then the relative formation dynamics are stabilized if and only if $F(z)$ stabilizes $L$ in the sense of Theorem 9 and $K(z)$ stabilizes $P(z)$.

Proof: We prove the presence of a separation principle through the use of a transformation of coordinates. The state-space equations of motion for the plant are given in (14). The predictor $\tilde{P}$ is presumed to be identical to the plant $P$, and has the same equations of motion with $x, y$ replaced by $\tilde{x}, \tilde{y}$. The information flow filter $R$ is defined in (20), but with the feedforward correction term added to the output, as discussed above. Letting the state-space representation of $R$ be $\left(R_{A}, R_{B}, R_{C}, R_{D}\right)$, we see that the state-space represntation of $H$ satisfies $H_{A}=R_{A}, H_{B}=R_{B}, H_{C}=-R_{C}, H_{D}=1-R_{D}$. Note that $H$ is stable due to the assumptions of Theorem 9. The states for the information flow laws and feedforward filters for all $N$ vehicles are $q$ and $r$, respectively. If one solves for the states, the resulting system of equations in terms of $\left(H_{A}, H_{B}, H_{C}, H_{D}\right)$ is $X_{k+1}=\Psi X_{k}$, where $X_{k}^{T}=\left[x_{k}^{T}, v_{k}^{T}, \tilde{x}_{k}^{T}, r_{k}^{T}, q_{k}^{T}\right]$ where $\Psi$ is defined in (26), as shown at the bottom of the page, in which $\hat{X}=I_{N} \otimes X, \phi=\hat{H}_{B}\left(\hat{P}_{D} \hat{K}_{D} \Delta+G\right)$, and $\Delta=\left(I_{N}-\hat{P}_{D} \hat{K}_{D}\right)^{-1}$, which is invertible by assumption of well-posedness of the interconnection. If we apply the transformation

$$
T=I_{N} \otimes\left(\begin{array}{lllll}
1 & 0 & 0 & 1 & 0 \\
0 & 0 & 1 & 0 & 0 \\
0 & 0 & 0 & 1 & 0 \\
0 & 1 & 0 & 0 & 1 \\
0 & 0 & 0 & 0 & 1
\end{array}\right)
$$

to the system matrix, we recover the matrix given in (28), as shown at the bottom of the next page. Stability of the system

$$
\Psi=\left(\begin{array}{ccccc}
\hat{P}_{A} & \hat{P}_{B} \Delta \hat{K}_{C} & \hat{P}_{B} \hat{K}_{D} \Delta \hat{P}_{C} & -\hat{P}_{B} \hat{K}_{D} \Delta \hat{H}_{C} & \hat{P}_{B} \hat{K}_{D} \Delta \hat{H}_{C} \\
0 & \hat{K}_{A}+\hat{K}_{B} \hat{P}_{D} \Delta \hat{K}_{C} & \hat{K}_{B} \Delta \hat{P}_{C} & -\hat{K}_{B} \Delta \hat{H}_{C} & \hat{K}_{B} \Delta \hat{H}_{C} \\
0 & \hat{P}_{B} \Delta \hat{K}_{C} & \hat{P}_{A}+\hat{P}_{B} \hat{K}_{D} \Delta \hat{P}_{C} & -\hat{P}_{B} \hat{K}_{D} \Delta \hat{H}_{C} & \hat{P}_{B} \hat{K}_{D} \Delta \hat{H}_{C} \\
0 & \hat{H}_{B} \hat{P}_{D} \Delta \hat{K}_{C} & \hat{H}_{B} \Delta \hat{P}_{C} & \hat{H}_{A}-\hat{H}_{B} \hat{P}_{D} \hat{K}_{D} \Delta \hat{H}_{C} & \hat{H}_{B} \hat{P}_{D} \hat{K}_{D} \Delta \hat{H}_{C} \\
\hat{H}_{B} L \hat{P}_{C} & \hat{H}_{B} \hat{P}_{D} \Delta \hat{K}_{C} & \phi \hat{P}_{C} & -\phi \hat{H}_{C} & \hat{H}_{A}+\phi \hat{H}_{C}
\end{array}\right)
$$



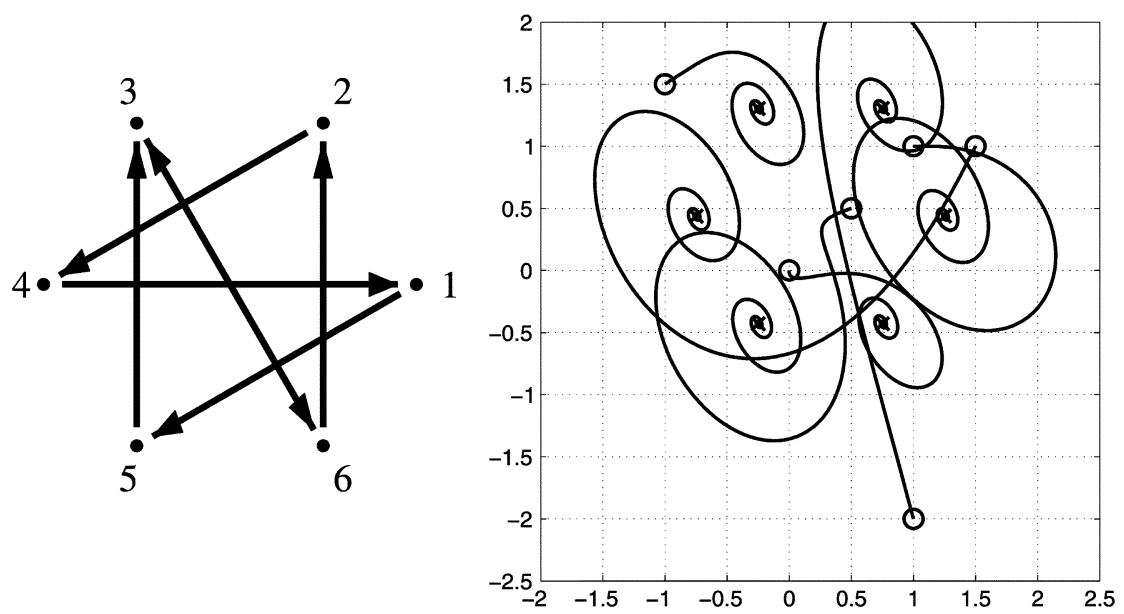

Fig. 7. Communication topology and hexagon acquisition using only sensed infromation.

is equivalent to stability of the blocks along the diagonal. The first, $P_{A}$, is neutrally stable by assumption. The assumption that the information flow law stabilizes the graph is equivalent to the second block, $H_{A}+H_{B} G H_{C}$, being stable. The third block along the diagonal, which comprises the third and fourth columns/rows, is stable if $K$ stabilizes $P$. (The reader will verify that this is the matrix derived when $K$ and $P$ are interconnected directly via feedback.) The final block represents the states of $H$, which is stable by the assumption in Theorem 9 .

Remarks: Equation (28) can be interpreted in the following way. The first set of states are open-loop copies of the vehicles' dynamics, and represent mismatches in initial conditions between the predictor and the actual vehicle. The second set is identical to the dynamics of (20), whose stability and convergence properties were studied previously. The output of this set of states acts as a reference for the $N$ copies of $P$ in feedback with $K$, found in the third and fourth rows. We see, therefore, that the effect of this architecture is to supply the local controllers with a reference signal that represents the error of that vehicle relative to a common reference trajectory whose dynamics obey the open-loop dynamics of an individual vehicle. The final set of states represent the feedfoward component. These states are unobservable in the motion of the vehicles, but are stable by design. For the case where $R(z)=1 / z$, we see that $H(z)=(z-1) / z$, which confirms our intuition that the role of the predictor is to compute the expected change in the vehicle's position and add it to the transmitted information, as a means of compensating for communication lags.

Several observations can be made regarding implementation. The first is that the motion of the formation is sensitive to mismatches between initial conditions of the vehicle and predictor. This can lead to drift of the cluster if the mismatch is in velocities. It should be possible to improve upon this through the use of an observer which will prevent the vehicle and predictor from diverging. Another solution is to initialize the predictor using earlier measurements. The trajectories of the vehicles will also depend on the ability of the information flow filter to track the natural motion of the vehicles. When the natural motion of the vehicles is at rest, we have seen that it achieves a proper steady state when $c=1$. When the natural motion is secular drift or oscillation (corresponding to poles at the origin or along the $j \omega$ axis), the quality of the reference signal will depend on the ability of the information flow filter to track signals at the relevant frequencies.

Example 3 (Formation Acquisition): We return to the example of the planar double integrator (now discretized in time), and consider the following problem. Six vehicles are required to acquire positions relative to one another such that they form the points of a regular hexagon. The assignment of points to vehicles has been predetermined, as has the communication graph, shown in Fig. 7. A SISO controller has been designed, and its stability checked using the tools of Section III-B. The initial positions of the vehicles are marked with "o" in Fig. 7, and their final positions with an "x." The initial velocities are all zero.

As can be seen in Fig. 7, the vehicles, while stable, follow circuitious trajectories before reaching equilibrium. This is due to two factors. The first is that each vehicle's initial motion attempts to place it in the correct position relative to the vehicles it can sense. However, those vehicles are themselves moving to new locations, causing the initial motions of each vehicle to be off target. The second reason is that the closed-loop formation dynamics have induced oscillations not anticipated in the initial design.

To implement the information flow paradigm, we use $F_{2}(z)$ from Example 2, and implement $H(z)$ accordingly. In this case, the vehicles follow the trajectories shown on the left in Fig. 8. The trajectories are smoother, but still show some curving due to action of the control law prior to convergence of the information

$$
T^{-1} \Psi T=\left(\begin{array}{ccccc}
\hat{P}_{A} & 0 & 0 & 0 & 0 \\
-\hat{H}_{B} L \hat{P}_{C} & \hat{H}_{A}+\hat{H}_{B} G \hat{H}_{C} & 0 & 0 & 0 \\
0 & -\hat{K}_{B} \Delta \hat{H}_{C} & \hat{K}_{A}+\hat{K}_{B} \hat{P}_{D} \Delta \hat{K}_{C} & \hat{K}_{B} \Delta \hat{P}_{C} & 0 \\
0 & -\hat{P}_{B} \hat{K}_{D} \Delta \hat{H}_{C} & \hat{P}_{B} \Delta \hat{K}_{C} & \hat{P}_{A}+\hat{P}_{B} \hat{K}_{D} \Delta \hat{K}_{C} & 0 \\
\hat{H}_{B} L \hat{P}_{C} & -\phi \hat{H}_{C} & \hat{H}_{B} \hat{P}_{D} \Delta \hat{K}_{C} & \hat{H}_{B} \Delta \hat{P}_{C} & \hat{H}_{A}
\end{array}\right)
$$



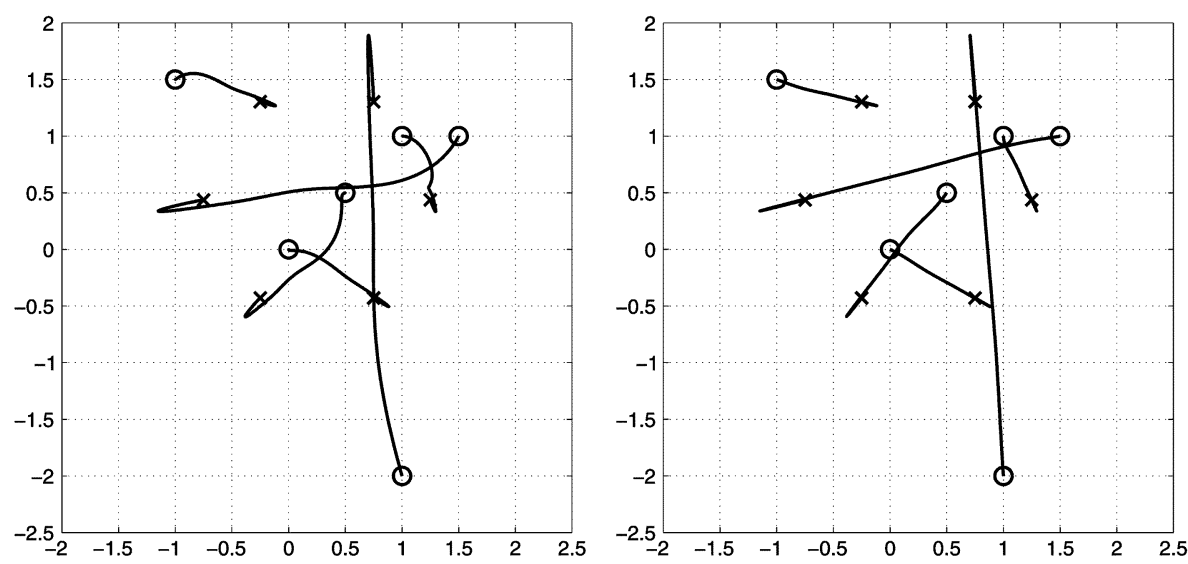

Fig. 8. Hexagon acquisition with information flow using (left) no information preconvergence and (right) one second of information preconvergence.
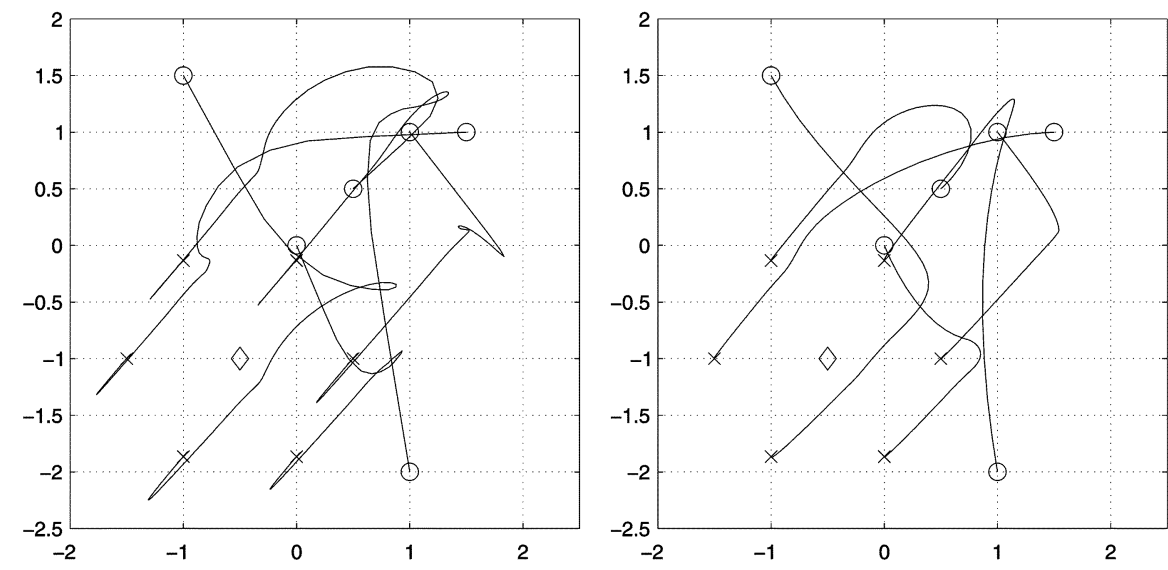

Fig. 9. Target acquisition with (left) no information flow and (right) information flow.

flow law. The right-hand figure shows the trajectories followed by the vehicles when the information flow law is enabled one second prior to enabling the control loop. In this case, the vehicles follow straight lines to their targets. Note that the formation center is identical in the two cases despite the differing trajectories. This is due to the decoupling of the information flow law from expected formation motion. Finally, note that the oscillations present in Fig. 7 have been eliminated due to the decoupling of vehicle dynamics from information flow dynamics.

Example 4 (Target Acquisition): While the information flow method was developed to handle relative motion, it can accommodate the case where a subset of the vehicles is measuring its position relative to a target. In this example, the goal of the formation is to position itself as a regular hexagon with the target in the center. To achieve this, the vehicles that see the target incorporate this relative position measurement as if it were a vehicle, and generate fictitious information to represent the target as a vehicle. Recalling that the information flow filter is meant to converge to each vehicle's relative error, the appropriate information for a target to transmit is zero- the target is already where it supposed to be relative to the desired formation location. In this case, the graph is not strongly connected (the target does not sense any vehicle), but it can be shown that the information flow law will still converge to the desired results; see [13] for more details.

Fig. 9 shows the formation evolution in a case where it begins to converge as in the previous example, and a single vehicle detects a target while acquiring the hexagon. In this example, a target becomes visible to a single vehicle as the formation is acquiring the hexagon. In the left-hand figure, which does not implement information flow, the lack of global information causes the formation to overshoot the target, marked with a diamond, and to slowly settle into the desired position. The right-hand plot shows the same situation with information flow enabled. In this case, the information flow loop disseminates the target information to the other vehicles, causing the information flow loop to treat the target as the formation leader and use its position as the common reference. In this case, the formation gracefully changes course and quickly acquires the target. Note that the introduction of the target causes the Laplacian itself to change while the formation is moving, but this does not preclude successful target acquisition.

The double integrator case has been worked as an extended example in this paper, but the methods works equally well for other cases. In [13], the case of relative stabilization of Hill's equations, which model relative satellite dynamics (see [1]) is explored to show how periodic orbits can be acquired using this method. Additional cases, such as changing commanded offsets, are explored in [13].

\section{CONCLUSION}

The information flow law proposed in Section IV shows the utility of the stability theorem in analyzing the behavior of vehicle formations and in synthesizing control solutions. We expect that this framework will be generally useful in analysis 
of formation stability problems and will be a useful starting point for future research. In particular, we expect that the qualitative insights into graph properties which are desirable from the perspective of stability can be quantified. A principle that allows each vehicle in the graph to determine its impact on formation stability using only local information would be particularly useful, as it could form the basis of a protocol for information weighting.

Our approach relies on two key ideas. The first is the use of dynamical systems as a paradigm for understanding information exchange between vehicles, and the design of a dynamical system which enables the vehicles to achieve consensus on the formation center. The second is the use of feedforward compensation to render the sensed and transmitted information timely. While this paper restricts its focus to linear systems with fixed time delays, we expect that this approach can be extended to nonlinear vehicle systems and systems with variable time delays. Nonlinear vehicles typically possess a center manifold which corresponds to the surface on which the vehicle performs locomotion; if the information flow is restricted to that surface, it should be possible to extend the information flow principle to that class of problems. We also conjecture that our approach can be extended to systems with variable time delays through appropriate extension of the feedforward term used to achieve stability separation. At the moment, the main limitation in the method is the constraint that $c=1$ in the information flow law. The need for consensus among vehicles forces the information flow law to be neutrally stable, which means that information never decays out. This renders the system sensitive to sensor errors and mismatches in initial conditions. One possibility for improvement is a protocol for resetting the information to zero periodically or in response to an event as a means of limiting any drift. Such a protocol could lie in a higher layer in the control architecture, and may itself require stability analysis. Alternate approaches to zeroing out accumulated error will be explored in the future.

\section{REFERENCES}

[1] R. Burns et al., "TechSat21: Formation design, control, and simulation," in Proc. IEEE Aerospace Conf., 2000, pp. 19-25.

[2] H. Schaub et al., "Spacecraft formation flying control using mean orbital elements," J. Astronaut. Sci., vol. 48, no. 1, pp. 69-87, 2000.

[3] L. E. Buzogany, M. Pachter, and J. J. d'Azzo, "Automated control of aircraft in formation flight," in Proc. AIAA Conf. Guidance, Navigation, and Control, 1993, pp. 1349-1370.

[4] J. D. Wolfe, D. F. Chichka, and J. L. Speyer, "Decetntralized controllers for unmanned aerial vehicle formation flight," in Proc. AIAA Conf. Guidance, Navigation, and Control, 1996, AIAA Paper 96-3833.

[5] T. B. Curtin, J. G. Bellingham, J. Catipovic, and D. Webb, "Autonomous oceanographic sampling networks," Oceanography, vol. 6, pp. 86-94, 1993.

[6] T. R. Smith, H. H. mann, and N. E. Leonard, "Orientation control of multiple underwater vehicles," in Proc. 40th IEEE Conf. Decision and Control, 2001, pp. 4598-4603.

[7] J. G. Bender, "An overview of systems studies of automated highway systems," IEEE Trans. Veh. Technol., vol. 40, pp. 82-99, Jan. 1991.

[8] D. Swaroop and J. K. Hedrick, "Constant spacing strategies for platooning in automated highway systems," ASME J. Dyna. Syst., Measure., Control, vol. 121, pp. 462-470, 1999.

[9] H. Yamaguchi, "A cooperative hunting behavior by mobile robot troops," Int. J. Robot. Res., vol. 18, no. 9, pp. 931-940, 1999.

[10] H. Yamaguchi, T. Arai, and G. Beni, "A distributed control scheme for multiple robotic vehicles to make group formations," Robot. Auton. Syst., vol. 36, no. 4, pp. 125-147, 2001.

[11] D. Yanakiev and I. Kanellakopoulos, "A simplified framework for string stability analysis in AHS," in Proc. 13th IFAC World Congr., vol. Q, San Francisco, CA, 1996, pp. 177-182.
[12] M. Egerstedt, X. Hu, and A. Stotsky, "Control of mobile platforms using a virtual vehicle approach," IEEE Trans. Automat. Contr., vol. 46, pp. 1777-1782, Nov. 2001.

[13] J. A. Fax, "Optimal and cooperative control of vehicle formations," Ph.D. dissertation, California Inst. Technol., Pasadena, CA, 2002.

[14] J. A. Fax and R. M. Murray, "Graph laplacians and stabilization of vehicle formations," in Proc. 15th IFAC Conf., 2002, pp. 283-288.

[15] _ , "Information flow and cooperative control of vehicle formations," in Proc. 15th IFAC Conf., 2002, pp. 283-288.

[16] J. Corfmat and A. Morse, "Decentralized control of linear multivariate systems," Automatica, vol. 12, pp. 476-495, 1976.

[17] D. D. Siljak, Decentralized Control of Complex Systems, ser. Mathematics in Science and Engineering. Boston, MA: Academic, 1990, vol. 184.

[18] J. Desai, J. P. Ostrowski, and V. Kumar, "Modeling and control of formations of nonholonomic mobile robots," IEEE Trans. Robot. Automat., vol. 17, pp. 905-908, June 2001.

[19] M. Mesbahi and F. Hadaegh, "Formation flying of multiple spacecraft via graphs, matrix inequalities, and switching," AIAA J. Guid., Control, Dyna., vol. 24, no. 2, pp. 369-377, 2001.

[20] P. Tabuada, G. J. Pappas, and P. Lima, "Feasible formations of multiagent systems," in Proc. Amer. Control Conf., 2001, pp. 56-61.

[21] J. F. Heagy, T. L. Carroll, and L. M. Pecora, "Synchronous chaos in coupled oscillator systems," Phys. Rev. E, vol. 50, no. 3, pp. 1874-1885, 1994.

[22] L. M. Pecora and T. L. Carroll, "Master stability functions for synchronized coupled systems," Phys. Rev. Lett., vol. 80, no. 10, pp. 2109-2112, 1998.

[23] C. W. Wu and L. O. Chua, "Synchronization in an array of linearly coupled dynamical systems," IEEE Trans. Circuits Syst. I, vol. 42, pp. 430-447, Aug. 1995.

[24] C. W. Wu, "Synchronization in arrays of coupled nonlinear systems: Passivity, circle criterion, and observer design," IEEE Trans. Circuits Syst. I, vol. 48, pp. 1257-1261, Oct. 2001.

[25] R. Diestel, Graph Theory, ser. Graduate Texts in Mathematics. New York: Springer-Verlag, 1997, vol. 173.

[26] R. Merris, "Laplacian matrices of graphs: A survey," Linear Alg. Applicat., vol. 197, 198, pp. 143-176, 1994.

[27] _ , "A survey of graph Laplacians," Linear Multilinear Alg., vol. 39, pp. 19-31, 1995.

[28] F. R. K. Chung, Spectral Graph Theory, ser. Regional Conference Series in Mathematics. Providence, RI: Amer. Math. Soc., 1997, vol. 92.

[29] A. Berman and R. J. Plemmons, Nonnegative Matrices in the Mathematical Sciences. New York: Academic, 1979.

[30] R. Horn and C. Johnson, Matrix Analysis. Cambridge, U.K.: Cambridge Univ. Press, 1985.

[31] R. S. Varga, Matrix Iterative Analysis, 2nd ed, ser. Springer Series in Computational Mathematics. New York: Springer-Verlag, 1991, vol. 27.

[32] K. Zhou and J. C. Doyle, Essentials of Robust Control. Upper Saddle River, NJ: Prentice-Hall, 1998.

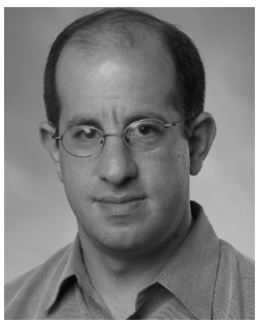

J. Alexander Fax (M'02) received the B.S.E. degree in mechanical and aerospace engineering from Princeton University, Princeton, NJ, in 1993, and the $\mathrm{Ph} . \mathrm{D}$. degree in control and dynamical systems from the California Institute of Technology, Pasadena, in 2002.

He is a Member of Technical Staff at Northrop Grumman Electronic Systems, Woodland Hills, CA. His areas of interest include navigation and control of autonomous vehicles, advanced navigation sensing technologies, and nonlinear control theory.

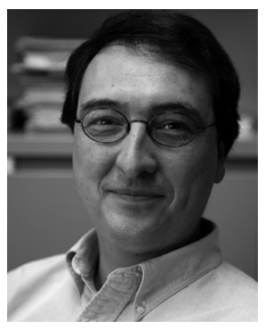

Richard M. Murray (S'83-M'85) received the B.S degree in electrical engineering from the California Institute of Technology, Pasadena, in 1985, and the M.S. and Ph.D. degrees in electrical engineering and computer sciences from the University of California, Berkeley, in 1988 and 1991, respectively.

$\mathrm{He}$ is currently a Professor of Mechanical Engineering and the Chair of the Division of Engineering and Applied Science, California Institute of Technology. His research is in the application of feedback and control to mechanical, information, and biolog- 